\title{
Is there a dissociative process in sleepwalking and night terrors?
}

\author{
D Hartman, A H Crisp, P Sedgwick, S Borrow
}

\begin{abstract}
The enduring and contentious hypothesis that sleepwalking and night terrors are symptomatic of a protective dissociative mechanism is examined. This is mobilised when intolerable impulses, feelings and memories escape, within sleep, the diminished control of mental defence mechanisms. They then erupt but in a limited motoric or affective form with restricted awareness and subsequent amnesia for the event. It has also been suggested that such processes are more likely when the patient has a history of major psychological trauma. In a group of 22 adult patients, referred to a tertiary sleep disorders service with possible sleepwalking/night terrors, diagnosis was confirmed both clinically and polysomnographically, and only six patients had a history of such trauma. More commonly these described sleepwalking/night terrors are associated with vivid dream-like experiences or behaviour related to flight from attack. Two such cases, suggestive of a dissociative process, are described in more detail.
\end{abstract}

The results of this study are presented largely on account of the negative findings. Scores on the dissociation questionnaire (DIS-Q) were normal, although generally higher in the small "trauma" subgroup. These were similar to scores characterising individuals with posttraumatic stress disorder. This "trauma" group also scored particularly highly on the anxiety, phobic, and depression scales of the Crown-Crisp experiential index. In contrast the "no trauma" group scored more specifically highly on the anxiety scale, along with major trends to high depression and hysteria scale scores. Two cases are presented which illustrate exceptional occurrence of later onset of sleepwalking/night terrors with accompanying post-traumatic symptoms during wakefulness. It is concluded that a history of major psychological trauma exists in only a minority of adult patients presenting with sleepwalking/night terror syndrome. In this subgroup trauma appears to dictate the subsequent content of the attacks. However, the symptoms express themselves within the form of the sleepwalking/night terror syndrome rather than as rapid eye movement sleep related nightmares. The main group of subjects with the syndrome and with no history of major psychological trauma show no clinical or DIS-Q evidence of dis- sociation during wakefulness. The proposition that, within the character structure of this group, the mechanism still operates but exclusively within sleep remains a possibility.

(Postgrad Med f 2001;77:244-249)

Keywords: sleepwalking; night terrors; dissociation; post-traumatic stress disorder

Sleepwalking and night terrors are related parasomnias, both characterised by sudden arousals from deep (polysomnographic stage 4) sleep during which the individual may present with a constricted awareness of his/her surroundings. In the case of sleepwalking, this arousal is associated with motor activity which may be elaborate and purposive. On the other hand the arousal of night terrors manifests as a terrified scream accompanied by intense autonomic discharge, and often motor activity which is typically stereotyped, perseverative, and less purposive than that of sleepwalking. ${ }^{12}$ In practice, these conditions are not distinct, but exist on a continuum as many individuals will have features of both. ${ }^{3}$ Night terrors are distinct from nightmares (which occur in rapid eye movement (REM) sleep when muscle tone is greatly reduced), but they bear some resemblance to other, more recently recognised, parasomnias. Thus, REM sleep behaviour disorder is characterised by complex motor activity similar to that of sleepwalking, but occurs in the context of REM rather than deep sleep. ${ }^{4}$ This condition is often precipitated by psychological trauma, and may be a variant of the post-traumatic nightmares described by Van der Kolk, et al ${ }^{\overline{ }}$ and Hefez et al. ${ }^{6}$ REM sleep behaviour disorder is characterised by unpleasant dreams occurring in both REM and non-REM sleep and often associated with motor activity. Van der Kolk and his colleagues speculated that the nightmares of posttraumatic stress disorder (PTSD) are intermediate between night terrors and classical nightmares, sharing features of both conditions.

Dissociation is regarded as a crude and primitive psychological defence which is utilised because of the inadequacy or failure of more mature and adaptive defences in the face of overwhelming stress. Dissociation may be regarded as maladaptive in that the distressing experience is encapsulated, separated from everyday consciousness, and resists integration with the individual's day-to-day experience. Van der Kolk and Van der Hart's useful account suggests that intense or prolonged trauma, particularly when associated with extreme physiological arousal and 
developmental immaturity, may resist psychic integration at a narrative or linguistic level. ${ }^{7}$ Such experiences are encoded in an enactive, that is, sensorimotor form, and persist largely out of conscious awareness as dissociative (or "somnambulistic") states. These may emerge as activity rather than narrative memory when prompted by internal or environmental cues. This process has been used as an explanatory model for the re-enactment of trauma which is seen in PTSD and multiple personality disorder.

PTSD is characterised by the following features: exposure to a traumatic event accompanied by intense fear or helplessness; persistent re-experiencing of the traumatic event (recurrent intrusive memories, recurrent distressing dreams, and distress and physiological arousal on exposure to cues that resemble aspects of the event); avoidance of stimuli associated with the trauma; constriction of general responsiveness with emotional numbing and detachment; and symptoms of increased arousal such as irritability, hypervigilance, or outbursts of anger. ${ }^{2}$

While there is a clear constitutional component to the aetiology of sleepwalking and night terrors ${ }^{8}$ there is also a long tradition of psychological theories about the causation of these conditions. Broughton ${ }^{1}$ and Fisher et al ${ }^{9}$ recognised that mentation does occur in stage 4 sleep, although these thoughts are soon forgotten, and the parasomnia may be the consequence of a particularly aversive thought occurring in this stage of sleep. It has been suggested that, in some cases, sleepwalking may be understood as a motoric re-enactment of a repressed traumatic experience. ${ }^{10}$ If sleepwalking and night terrors do arise as a response to arousal producing thoughts in stage 4 sleep, perhaps related to traumatic memories, then it would be plausible that these parasomnias reflect one variant of a dissociative process, in which intolerable experiences are still defended against by being isolated in a dissociated sphere of consciousness ${ }^{11} 12$ within the acutely aroused state that has interrupted sleep. Crisp has recently suggested that the eruption of sleepwalking and night terrors from deep sleep may be a function of the profound resting state of the forebrain and the associated resting of the usual waking mental defence mechanisms, for example selective denial and repression, which protect the individual from such experience during normal wakefulness. ${ }^{13}$

In this study we examine the hypothesis that sleepwalking and night terrors take place by means of a dissociative mechanism. It supposes that, if this mechanism is operating as a feature of the attack, it may also be present as a mental characteristic of normal wakefulness. This question is elaborated into three more specific questions. Firstly, can one find qualitative evidence of a dissociative process within normal wakefulness in individuals suffering from sleepwalking and night terrors? Secondly, how frequent is a history of psychic trauma in patients presenting with sleepwalking/night terrors? Finally, are there clinical similarities between patients with sleepwalking/night terrors and individuals suffering from the dissociative disorders that can arise within PTSD?

\section{Method}

The study was carried out at a sleep disorders clinic within an NHS psychiatric service. The clinic was a national referral centre for sleepwalking and night terrors, and attracted adult patients who generally had a long history of severe sleepwalking or night terrors, often complicated by injury to themselves or their bed partners. The inclusion criteria were polysomnographic evidence (sudden awakenings from slow wave sleep) of sleepwalking or night terrors during at least one of two consecutive nights of polysomnography with infrared video monitoring, and compliance with Diagnostic and Statistical Manual of Mental Disorders, fourth revision (DSM-IV) ${ }^{2}$ criteria for night terrors ("sleep terror disorder") or sleepwalking. Twenty two consecutive referrals meeting these criteria were subjected to a detailed psychiatric assessment and where possible a bed partner or other informant was also interviewed. Particular attention was paid to the phenomenology of the parasomnia, and to any history of a traumatic experience, using the DSM-IV standard of severe trauma outside the range of normal human experience. Patients were also asked to complete two psychometric questionnaires, the dissociation questionnaire (DIS-Q), and the Crown-Crisp experiential index (CCEI). The DIS-Q was originally developed to measure the characteristic symptoms of proposed dissociative disorders such as multiple personality disorder, depersonalisation disorder and psychogenic amnesia, but also obtains high scores from individuals with PTSD and eating disorders. The instrument's validity and reliability are well established. ${ }^{14}$ In this paper we did not report the scores on the subscales of identity confusion, loss of control, amnesia, and absorption but we used the total DIS-Q score as an index of the severity of dissociative experiences during wakefulness. The results obtained from the DIS-Q were compared with reference values for "normals" and PTSD patients derived from Vanderlinden. ${ }^{14}$ The CCEI is a self report questionnaire measuring the constructs of anxiety, phobic anxiety, obsessionality, somatisation, depression and hysteria, the score range is $0-16$ on each scale, which has been extensively validated in various clinical and community samples. ${ }^{15}$ This was used as a broad measure of psychopathology and defensive style, and the CCEI results were compared with data matched for age and sex from the earlier population studies and similar comparative studies of parasomniacs ${ }^{11}$ using this instrument. The above comparisons were carried out by means of two sample $t$ tests. Satterthwaite's approximation to the degrees of freedom was used when the variances in the two groups were unequal. Tests were deemed significant at the $5 \%$ level. 


\section{Results}

Of the 22 subjects, 10 were ascribed a diagnosis of sleepwalking and 12 one of night terrors. The two groups are combined and examined together in this study; 10 subjects were male and 12 were female. The median age of onset of the disorder was 8 years (semi-interquartile range 12.5 years). The median age at referral to our adult sleep disorders secondary/tertiary referral service, was 35.5 years (semiinterquartile range 9 years). Six (27\%) of the 22 subjects gave an account, within a lengthy systematic history taking session, of significant earlier psychologically traumatic experiences: in most cases this took the form of severe abuse or neglect during childhood. Examples are given in the case reports below.

When patients with a history of trauma were compared to those without, they were very similar with regard to age of onset, age of referral, sex, and diagnosis (that is sleepwalking or night terrors). The "trauma" group differed from the "no trauma" group with regard to the phenomenology of the parasomnia. Of those who had a history of trauma, five out of six $(83 \%)$ reported vivid, dream-like mental content accompanying the event, as compared with four out of $16(25 \%)$ of those without a history of trauma $(p=0.049$ using Fisher's exact test). All the former reported attempting to flee from an attacker, compared with just $25 \%$ of the latter ( $p=0.003$, Fisher's exact test). Other themes reported by the "no trauma" group included searching for a lost object, searching for or consuming food, protecting a bed partner, or behaviour or vocalisation with no clear meaning. Two of the six who had experienced a traumatic event described mental content during the sleepwalking/night terrors which was an exact re-experiencing of the original event as now reported.

DIS-Q RESULTS (TABLE 1$)^{14}$

The "trauma" and "no trauma" groups were not significantly different from the normal reference data with regard to the DIS-Q total score. The "no trauma" group very closely resembled the normal group and scored highly significantly lower $(\mathrm{p}=0.000, t=-9.27$; $\mathrm{df}=32$ ) than the PTSD group. The "trauma" group scored substantially higher than normals (but not significantly different; Satterthwaite's correction used), and closer to the scores of the PTSD comparison group (again not significantly different).

CCEI RESULTS (TABLE 2$)^{12}$

The "no trauma" group scored significantly higher than the control group of matched normals on anxiety $(t=3.40 ; \mathrm{df}=365 ; \mathrm{p}=0.001)$, with trends for higher scores for depression $(t=$

Table 1 DIS-Q; results are mean (SD) total score

\begin{tabular}{lllll}
\hline Parasomnias & & & \multicolumn{2}{l}{ Comparisons $^{*}$} \\
\cline { 1 - 3 } \cline { 5 - 6 } $\begin{array}{l}\text { "No trauma” } \\
n=16)\end{array}$ & $\begin{array}{l}\text { "Trauma” } \\
(n=6)\end{array}$ & & $\begin{array}{l}\text { Normals } \\
(n=378)\end{array}$ & $\begin{array}{l}\text { PTSD } \\
(n=73)\end{array}$ \\
\hline $1.6(0.4)$ & $2.3(1.2)$ & & $1.5(0.4)$ & $2.9(0.7)$ \\
\hline
\end{tabular}

*Vanderlinden $1993 .^{14}$
Table 2 CCEI scale scores (range 0-16); results are mean (SD)

\begin{tabular}{llrr}
\hline & \multicolumn{2}{l}{ Parasomnias } & \\
\cline { 2 - 3 } & $\begin{array}{l}\text { "No trauma" } \\
(n=16)\end{array}$ & $\begin{array}{l}\text { "Trauma" } \\
(n=6)\end{array}$ & $\begin{array}{l}\text { Normals* } \\
(n=352)\end{array}$ \\
\hline Anxiety & $8.0(3.7)$ & $12.0(2.6)$ & $4.6(3.8)$ \\
Phobia & $3.3(2.7)$ & $6.5(3.5)$ & $4.0(2.9)$ \\
Obsessionality & $7.1(3.3)$ & $7.7(3.4)$ & $6.3(3.1)$ \\
Somatisation & $4.1(3.0)$ & $8.8(5.5)$ & $3.5(2.9)$ \\
Depression & $4.0(2.3)$ & $9.3(3.3)$ & $2.8(2.6)$ \\
Hysteria & $5.3(3.7)$ & $4.0(2.5)$ & $3.9(3.1)$ \\
\hline
\end{tabular}

${ }^{\star}$ Crisp et al, 1990 (352 subjects randomly selected from the general population, male and female, aged 17-44 years (mean (SD) age $30.8(8)$ years). ${ }^{12}$

$1.76 ; \mathrm{df}=365 ; \mathrm{p}=0.08)$ and hysteria $(t=1.76$; $\mathrm{df}=365 ; \mathrm{p}=0.084)$. The "trauma" group scored significantly higher than the control group of normals on the anxiety $(t=4.75 ; \mathrm{df}=$ $356 ; \mathrm{p}=0.000)$, phobia $(t=2.09 ; \mathrm{df}=356 ; \mathrm{p}=$ $0.038)$, and depression $(t=6.07 ; \mathrm{df}=356 ; \mathrm{p}=$ $0.000)$ subscales and with a trend towards a higher score on somatisation $(t=2.39$; $\mathrm{df}=5$; $\mathrm{p}=0.063)$ with Satterthwaite's approximation being used for this last analysis.

Two case examples are described in more detail, with information derived from the psychiatric assessments and psychometric questionnaires. These cases are not representative of the 22 subjects of the study, but were selected because they were typical of the "trauma" subgroup and demonstrated a plausible process linking life experiences and subsequent symptoms. Some biographical details have been changed to ensure anonymity.

\section{CASE REPORTS}

Case 1

Case 1 was a 31 year old unmarried women who lived with her elderly parents. She presented with a complaint of "nightmares" of five years' duration, and described them as follows: she would get up and walk in her sleep, generally after 60-90 minutes of sleep, and would often leave the house in her nightclothes. On a number of occasions she had driven her car several miles in this condition, and had come to full awareness in an isolated place, to the sound of her car alarm some distance away. These events were accompanied by vivid, terrifying, and stereotyped mental content in which she was attempting to fight off a male attacker. This "nightmare" was a re-enactment of an incident which occurred when she was 10 years old. While out walking, she had been abducted, taken to a shed, and raped in a sadistic manner by a man not known to her. He threatened to maim her if she spoke of it, and when she returned home she concealed her internal injuries, and "put it to the back of my mind". She completed school and went on to train as a nurse. She remained heterosexually inactive, and was stable and successful in other respects. She had no psychiatric problems until the onset of her presenting symptom, five years earlier. This was precipitated abruptly by the experience of nursing a young girl who had also been raped and beaten.

On psychiatric assessment, she fulfilled the DSM-IV criteria ${ }^{2}$ for a major depressive episode of moderate severity and also for 
delayed onset PTSD. On the CCEI she had a moderately raised depression score. She scored within the normal range on all subscales of the DIS-Q.

Case 2

Case 2 was a 40 year old married man with two children, referred with a complaint of "nightmares" since childhood, which had become much worse over the previous two years. In these events he would see snakes in his bedroom, or feel that he was being pursued by an evil presence; he would then awaken in a state of extreme terror and confusion. Over the previous 18 months he had started to demonstrate dangerous behaviour during these episodes, for example wandering considerable distances outside the house in his pyjamas or naked, or hitting his wife in bed. These events were said to take place within an hour or two of him going to sleep, at between 2 and $3 \mathrm{am}$. $\mathrm{He}$ also described bizarre experiences during his waking hours: auditory, visual, and olfactory hallucinations which resembled his "nightmares", a sense that he was in telepathic communication with his mother, and a sense that he had more than one person inside him. He had periods of amnesia and subsequently discovered that he had carried out some bizarre activity. As a child he was severely beaten by his father and mother who also sexually abused him. He married at the age of 20 and had two children. He had no contact with psychiatric services until three months before presentation to the sleep service, when he had a brief admission for depressive symptoms. This was precipitated by disclosures that one of his children had been sexually abused.

On assessment this patient fulfilled DSM-IV criteria $^{2}$ for major depressive episode of moderate severity, and had symptoms suggestive of multiple personality disorder. On the CCEI he had markedly raised scores on the anxiety, depression, somatisation, and obsessionality subscales, and on the DIS-Q he had markedly raised scores on all subscales, compatible with a diagnosis of multiple personality disorder.

\section{Discussion}

Our clinic attracts nationwide referrals of patients with sleepwalking and night terrors, and the patients studied were therefore probably unrepresentative of sleepwalking and night terrors in the general population. They were probably more representative of adult sleepwalking or night terror sufferers who had recently required attention from primary care services and sometimes from other psychiatric or neurological services because of the severity or hazardous nature of their symptoms. All 22 subjects we report on here yielded polysomnographic confirmation of the diagnosis. Six of them gave a history of previous severe psychological trauma, and this generally occurred in childhood. While this was a clinically substantial proportion, it was distinctly less than in the reported prevalence of childhood trauma among patients with dissociative disorders or among psychiatric patients in general. ${ }^{16}$ This does not support the hypothesis that sleepwalking/night terrors is generally the result of a dissociative response to trauma. However, when the group of patients with sleepwalking/night terrors with a history of psychological trauma was examined separately, subjects appeared to show distinctive characteristics. Sleepwalking and night terrors are generally described as being associated with limited or no mental content during the events, ${ }^{2}$ and this feature is commonly used to distinguish them clinically from true nightmares. However, in this study a majority of patients with a history of trauma described vivid, nightmare-like experiences in association with some of their sleepwalking or night terror events. Our patients in the "trauma" group were also more likely to experience sleepwalking or night terror events in which the subjective experience and overt behaviour was one of being attacked and attempting to escape or fight back, which invites a search for meaningful links between symptoms and past experience. This was graphically suggested by the two individuals who re-experienced the trauma during the sleepwalking and night terror episodes (cases 1 and 2). Van der Kolk et al describe similar events among some PTSD sufferers, ${ }^{5}$ but it is not clear whether these should be regarded as atypical nightmares, atypical sleepwalking/night terror events, or as distinct parasomnias, as suggested by Ross and Morrison. ${ }^{17}$ To exclude PTSD sufferers from a study of sleepwalking and night terrors because of their anomalous clinical qualities, as was done by Hartmann et al without the benefit of polysomnographic evaluation, ${ }^{18}$ seems undesirable to us. It ignores the possibility that PTSD sufferers with sleepwalking/night terrors are merely at one end of the symptomatic spectrum of night terrors. Such outliers may well have explanatory value for the broad spectrum of the disorders in question.

It is possible that some individuals in the non-trauma group had had dramatic experiences, no longer in the forefront of their minds, but which were meaningfully related to their sleepwalking/night terrors, and that aspects of these were being expressed in their sleeping behaviours; such cases are described, for example, by Sours. ${ }^{11}$ In an earlier paper we have described how frantic searching behaviour within a childhood onset of sleepwalking appeared to relate to death of a father shortly beforehand. ${ }^{13}$

The results of the psychometric investigations are suggestive rather than conclusive, especially in view of the multiple testing. With regard to the CCEI, the patients with a history of a traumatic experience have generally higher scores, suggesting overall higher levels of psychopathology and distress. The high depression score is notable but the mean levels of anxiety and phobia scores are especially high and probably consistent with the very high levels of arousal said to be a feature of PTSD. ${ }^{2}$ In contrast, the "no trauma" group scored highly only on the anxiety scale of the CCEI and, to a lesser extent, on the depression and hysteria scales. The DIS-Q characteristically obtains high scores in conditions such as multiple 
personality disorder, borderline personality disorder and PTSD. ${ }^{14}$ The results in this study were inconclusive with regard to dissociation during waking hours as measured by the DIS-Q. Although differences did not reach statistical significance, there was a pattern in which the "trauma" group resembled PTSD subjects more than normals, while the "no trauma" group closely resembled the normal reference values.

The cases described illustrate the relationship between sleepwalking/night terror phenomenology and symptoms reported in the CCEI and DIS-Q. In cases 1 and 2 there were histories of childhood physical and sexual trauma. The motoric re-enactment of the circumstances of the trauma is suggestive of the encapsulated repetition described in $\mathrm{PTSD}^{2}$ and also seems to be a classical example of the dissociative process as originally described by Pierre Janet. ${ }^{7}$ Case 1 suffers from moderate depressive symptoms but scores within the normal range on all subscales of the DIS-Q. Case 2 suffers from significant psychiatric morbidity including possible multiple personality disorder in his waking hours and has markedly raised scores on all subscales of the DIS-Q.

In both cases the development of overt psychiatric symptoms, accompanied by dramatic sleepwalking, resulted from a secondary experience in adulthood which served to draw the individual's attention back to the original injury. In case 1 (who experienced acute trauma in childhood rather than the chronic trauma of case 2) these dissociative events were confined to her sleeping hours and she could tolerate her waking distress and depression. On the other hand, case 2 experienced sleepwalking as only one of a wide range of dissociative phenomena throughout the day and night, which may reflect the severity of his own psychic injury.

We would suggest that in certain traumatised patients dissociative mechanisms may come into play within the attack as part of their response to the intense arousal from slow wave sleep associated with distress and/or behaviours normally kept in check. Eruptions at this time may be facilitated by the state of the brain within deep sleep wherein blood supply to the cortex is minimal and related cerebral activity, including their usual mental defensive strategies, is in abeyance. ${ }^{13}$ Primitive sensorial restriction, involving the dissociative mechanism, may then come into play as a measure necessary to protect the individual's wakeful awareness under these circumstances and until such times as mental "order" can be restored. During such episodes the individual may be considered to be awake rather than asleep, but no more fully conscious of themselves than the daytime wakeful individual with an alleged dissociative mechanism operating to produce an amnesia, or the various expressions of multiple personality disorder.

Such a notion suggests a role for psychodynamic psychotherapy as proposed, practised, and reported by Kales et al, ${ }^{19}$ Calogeras, ${ }^{10}$ Sours, ${ }^{11}$ and Vela-Beuno and Soldatos. ${ }^{3}$ Drugs as advocated by Schenck et $a l^{20}$ are, in our experience, not very effective except for benzodiazepines but these bring their own addictive problems in such chronic conditions.

Thus, just over a quarter of sleepwalking/ night terror sufferers who have filtered through to a tertiary referral sleep disorders service report the experience of some degree of dissociative phenomena, as measured by the DIS-Q, during their waking lives, and also have a history of significant childhood psychological trauma. This group is also characterised by higher levels of psychological distress in general. However, these cases are the exceptions when we consider the patient group as a whole. The majority of our patients reported normal levels of dissociative process within normal wakefulness as measured by DIS-Q, and did not report a history of psychological trauma. One could speculate that if sleepwalking/night terrors is indeed a successful form of defensive dissociation, then the low prevalence of trauma in our study group might reflect "false negatives" in our history taking, as the subjects were successfully excluding painful memories from awareness. One possible limitation of this study is the assumption that dissociative experiences during wakefulness are a reliable index of the postulated dissociative process during sleep. It remains possible that a dissociative mechanism, precipitated by the sudden intense arousals that escape into the undefended brain in this syndrome within sleep, may still operate at that time. This could then explain both the sensorial deficit that characterises the syndrome and also the histrionic sense of themselves that these subjects often report (on the hysteria scale of the CCEI both in our earlier study and again, though to a lesser extent, in this one) but do not display within normal wakefulness.

Despite our reservations we report this study because we can find no other accounts of similar psychometric investigations of this disorder in adults in the literature. Adult patients with this syndrome do not readily present themselves, having ceased to complain or seek help after a succession of failed treatments earlier in life. Future studies are needed, combining polysomnography with functional brain imaging techniques and standardised diagnostic questionnaires for post-traumatic and dissociative disorders.

1 Broughton R. Sleep disorders: disorders of arousal? Science 1968;159:1070-8.

2 American Psychiatric Association. Diagnostic and statistical manual of mental disorders. 4th Ed. Washington, DC: APA, manual

3 Vela-Bueno A, Soldatos CR. Parasomnias: sleepwalking, night terrors, and nightmares. Psychiatry Annals 1987;17: $465-9$

4 Schenck C, Hurwitz T, Mahowald M. REM sleep behaviour disorder. Am f Psychiatry 1988;145:65.

5 Van der Kolk B, Blitz R, Burr W, et al. Nightmares and trauma: a comparison of nightmares after combat with lifelong nightmares in veterans. Am f Psychiatry 1984;141:18790.

6 Hefez A, Metz L, Lavie P. Long-term effects of extreme situational stress on sleep and dreaming. Am $\mathcal{f}$ Psychiatry 1987;144:344-7.

7 Van der Kolk B, Van der Hart O. Pierre Janet and the breakdown of adaptation in psychological trauma. Am f Psychiatry 1989;146:1530-40. 
8 Kales A, Soldatos C, Bixler E, et al. Hereditary factors in sleepwalking and night terrors. Br $\mathcal{F}$ Psychiatry 1980;137: 111-18.

9 Fisher C, Kahn E, Edwards A, et al. A psychophysiological study of nightmares and night terrors. III. Mental content and recall of stage 4 night terrors. F Nerv Ment Dis 1974;158:174-88.

10 Calogeras RC. Sleepwalking and the traumatic experience. Int f Psychoanal 1982;63:483-9.

11 Sours JA. Somnambulism. Its clinical significance and dynamic meaning in late adolescence and adulthood. Arch Gen Psychiatry 1963;9:112-25.

12 Crisp AH, Matthews BM, Oakey M, et al. Sleepwalking, night terrors, and consciousness. BMF 1990;300:360-2.

13 Crisp AH. The sleepwalking/night terror syndrome in adults. Postgrad Med F 1996;72:599-604.

14 Vanderlinden J. Dissociative experiences, trauma and hypnosis. Delft: Eburon, 1993.
15 Crown S, Crisp AH. Manual of the Crown-Crisp experiential index. London: Hodder \& Stoughton, 1979.

$16 \mathrm{Chu}$ JA, Dill DL. Dissociative symptoms in relation to childhood physical and sexual abuse. Am $\mathcal{F}$ Psychiatry 1990; 147:887-92.

17 Ross RJ, Morrison AR. Revising the differential diagnosis of the parasomnias in DSM-IIIR. Sleep 1989;12:287-9.

18 Hartmann E, Greenwald D, Brune P. Night terrorssleepwalking: personality characteristics. Sleep Research 1982;11:121.

19 Kales JD, Cadieux RJ, Soldatos CR, et al. Psychotherapy with night terror patients. Am f Psychother 1982;36:399407.

20 Schenck CH, Milner DM, Hurwitz TD, et al. Dissociative disorders presenting as somnambulism. Polysomnographic, video and clinical documentation (8 cases). Dissociation 1989;2:194-204.

\section{Medical Anniversary}

\section{Ernest Besnier, 21 April 1831}

Ernest Besnier (1831-1909) was born in Honfleur, France and became an internist. In 1873 he succeeded Bazin as dermatologist at St Louis Hospital, Paris, where his statue may be found. He first presented "lupus pernio de la face-synovites fongueuses (scrofulo-tuberculeuses) symmetriques des extremities superieures" at a weekly conference at that hospital. $\mathrm{He}$ referred to Hutchinson's patient, John W, but the distribution of the lesions was sufficiently dissimilar to justify his opinion that the two conditions were not identical.

Besnier was elected to the Academy of Medicine in 1881 as hygienist because of his contributions to epidemiology. In 1887 he published various articles on leprosy. $\mathrm{He}$ built histopathology and parasitology laboratories at St Louis Hospital, and we owe to him the term and technique of skin biopsy. He translated Kaposi's textbook Pathology and Treatment of Skin Diseases into French and wrote widely on a variety of skin disorders. He died in Paris on 15 May 1909, aged 78 years. Soon afterwards Tenneson provided histological confirmation of sar-

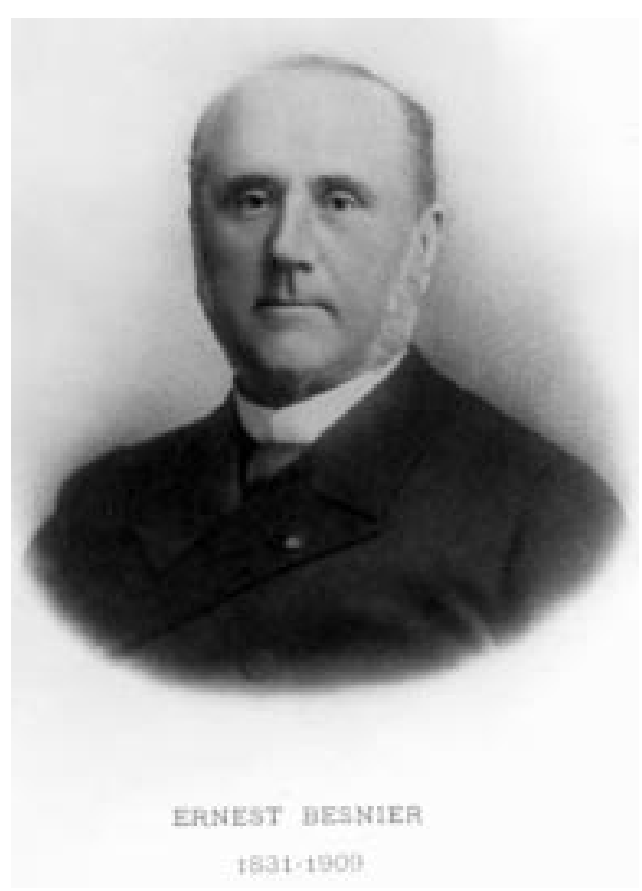
coid tissue in lupus perio. $-D$ G fames 